\title{
15
}

\section{Characterization of Mechanical Properties of Biological Tissue: Application to the FEM Analysis of the Urinary Bladder}

\author{
Eugenio Oñate ${ }^{a}$, Facundo J. Bellomo ${ }^{b}$, Virginia Monteiro ${ }^{a}$, Sergio Oller ${ }^{a}$, \\ and Liz G. Nallim ${ }^{b}$ \\ ${ }^{a}$ International Center for Numerical Method in Engineering (CIMNE), Technical \\ University of Catalonia, Spain \\ ${ }^{b}$ INIQUI (CONICET), Faculty of Engineering, National University of Salta, Argentina
}

\subsection{Introduction}

This chapter presents an approach for the mechanical behavior of soft biological tissue using the finiteelements method (FEM) and a general constitutive model. Specifically, we analyze the mechanical behavior of a urinary bladder starting from a procedure for obtaining the mechanical characterization of the biological tissue. The difficulty in this study lies not only in modeling the mechanical behavior of the bladder subjected to inflation under the presence of an internal fluid, but also in the difficulty encountered in determining the biomechanical properties of the biological tissue that forms the bladder. Bladder tissue is modeled as a composite material formed by soft matrix reinforced with preferentially oriented fibers. In the first part of the chapter we present a procedure for identifying the mechanical properties of biological tissue's main constituents by an inverse method. Then this information is used for the numerical simulation of the mechanical behavior of the bladder within the FEM.

The formulation can be applied to various types of biological tissues, both in the field of material characterization and in the numerical simulation of the tissue's biomechanical behavior. The approach presented in this chapter has been applied to the study of the arterial tissue behavior [1].

Multiscale Simulations and Mechanics of Biological Materials, First Edition. Edited by Shaofan Li and Dong Qian. (C) 2013 John Wiley \& Sons, Ltd. Published 2013 by John Wiley \& Sons, Ltd. 


\subsection{Inverse Approach for the Material Characterization of Biological Soft Tissues via a Generalized Rule of Mixtures}

There are few experimental data related to the mechanical properties of the main load-bearing constituents of soft biological tissues (collagen, elastin). Veseley [2] estimated the relative contribution of elastin to the mechanics of the porcine aortic valve. Roeder et al. [3] studied the structural-mechanical relationship of three-dimensional type I collagen matrices prepared in vitro. Holzapfel [4] presented a complete review of the biomechanical role of collagen in arterial walls. There are many difficulties associated with the isolation and testing of the individual components of soft tissues. For these reasons, experimental studies on mechanical properties of collagen are mainly related to tendons and ligaments [5-8]. On the other hand, several experimental studies had been carried out regarding the mechanical properties of extracellular matrix (ECM) scaffolds [9-16], derived mostly from porcine small intestinal submucosa and the urinary bladder. These scaffolds have become widely studied recently for a number of applications, including repair of the urinary bladder, esophagus, and myocardium [17]. These studies provide a very useful insight into the mechanics, fiber alignment, and kinematics of the ECM material.

A comprehensive study of soft tissues requires an adequate knowledge of the mechanical properties of their components. In this chapter a constitutive model based on a generalized rule of mixtures is proposed. The tissue is modeled as a biological composite material reinforced by families of collagen fibers. The material parameters of the model are identified by an inverse method. The application of this methodology leads to the mechanical characterization of matrix and collagen reinforcement. The model described in the following sections has been implemented in a finite-element formulation and applied to the mechanical analysis of the urinary bladder

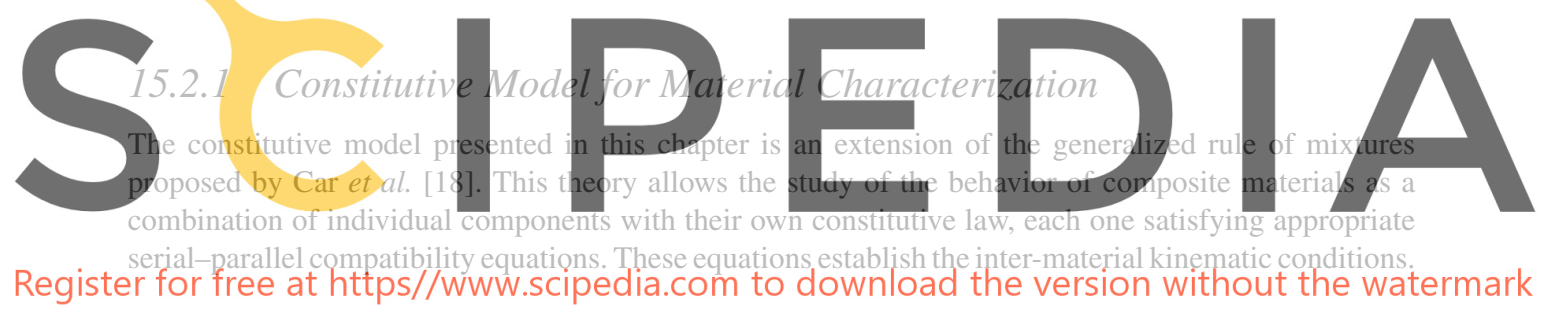

Generalized Rule of Mixtures for Finite Strains

The free energy in the reference configuration is given by

$$
m \Psi=\sum_{c=1}^{n} m_{c} k_{c} \Psi_{c}
$$

where $m$ and $m_{c}$ are, respectively, the composite mass and the $c$ th component mass and $\Psi$ and $\Psi_{c}$ are, respectively, the composite and the $c$ th component free energy. The Green-Lagrange strain tensor of the $c$ th component on the reference configuration is given by [19]

$$
\boldsymbol{E}_{c}=\left[\left(1-\chi_{c}\right) \boldsymbol{I}_{4}+\chi_{c} \boldsymbol{\phi}_{c}\right]: \boldsymbol{E},
$$

where $\boldsymbol{E}$ is the composite Green-Lagrange strain tensor and $\left(\phi_{i j k l}\right)_{c}$ is the serial behavior tensor in the reference configuration given by

$$
\left(\phi_{i j k l}\right)_{c}=\left(\frac{\partial^{2} \Psi_{c}}{\partial\left(E_{i j}\right)_{c} \partial\left(E_{r s}\right)_{c}}\right)^{-1}:\left[\sum_{c=1}^{n} k_{c}\left(\frac{\partial^{2} \Psi_{c}}{\partial\left(E_{r s}\right)_{c} \partial\left(E_{k l}\right)_{c}}\right)^{-1}\right]^{-1},
$$


where $\left(\partial^{2} \Psi_{c} / \partial\left(E_{i j}\right)_{c} \partial\left(E_{r s}\right)_{c}\right)^{-1}$ is the constitutive tensor of the $c$ th component and $\left[\sum_{c=1}^{n} k_{c}\left(\partial^{2} \Psi_{c} /\right.\right.$ $\left.\left.\partial\left(E_{r s}\right)_{c} \partial\left(E_{k l}\right)_{c}\right)^{-1}\right]^{-1}$ is the composite serial constitutive tensor, both expressed in the reference configuration. The fourth-order tensor $\boldsymbol{\phi}_{c}$ maps the strain to its serial counterpart in the reference configuration ensuring the serial equilibrium constraint.

The second Piola-Kirchhoff stress tensor can be obtained as follows:

$$
\boldsymbol{S}=m \frac{\partial \Psi}{\partial \mathbf{E}}=\sum_{c=1}^{n} k_{c} m_{c} \frac{\partial \Psi_{c}}{\partial \boldsymbol{E}_{c}} \frac{\partial \boldsymbol{E}_{c}}{\partial \boldsymbol{E}}=\sum_{c=1}^{n} k_{c} \mathbf{S}_{c} \frac{\partial \boldsymbol{E}_{c}}{\partial \boldsymbol{E}} .
$$

For further applications it is useful to write the Cauchy stress via a push-forward operation

$$
\sigma=\frac{1}{J} \mathbf{F} \cdot \mathbf{S} \cdot \mathbf{F}^{\mathrm{T}}
$$

where $\mathbf{F}$ is the deformation gradient.

In order to consider near incompressibility in soft issues, the energy is split into its isochoric and volumetric parts as follows:

$$
\Psi=\Psi_{\mathrm{vol}}(J)+\Psi_{\text {iso }}(\overline{\mathbf{C}}),
$$

where $\overline{\mathbf{C}}$ is the deviatoric part of the Cauchy-Green rigth tensor $\mathbf{C}$. The volumetric part of the free energy accounts for the tissue fluids' contribution, which is mainly responsible for the tissue quasiincompressibility. The interstitial tissue pressure is considered to be the same for all the solid phases, while the serial-paralle behavior
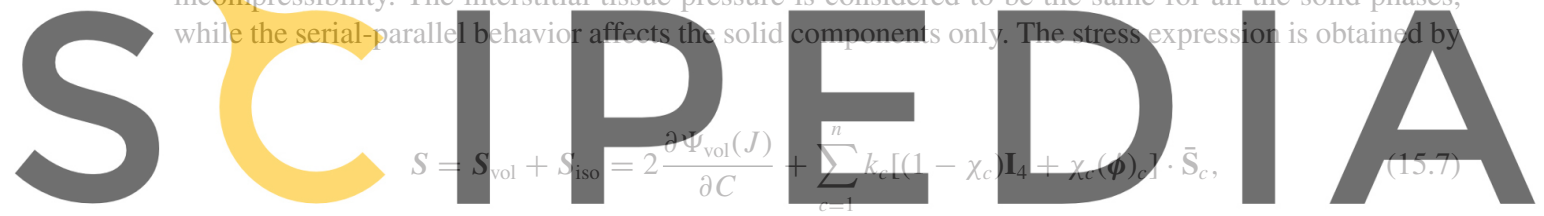

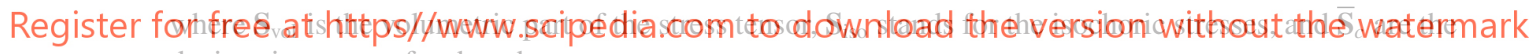
deviatoric stresses for the $c$ th component.

\section{Mixing Rule for the Uniaxial Case: A Discrete and Incremental Approach}

The inverse method proposed in this chapter employs results from uniaxial tests [20]. The uniaxial stress field is deduced from the general expressions (15.4) and (15.7) as

$$
\mathbf{S}^{\theta}=\mathbf{S}_{\mathrm{vol}}+\sum_{c=1}^{n} k_{c} \overline{\mathbf{S}}_{c} \frac{\partial \mathbf{E}_{c}}{\partial \mathbf{E}}=\mathbf{S}_{\mathrm{vol}}+\sum_{c=1}^{n} k_{c} \overline{\mathbf{S}}_{c} \frac{\partial}{\partial \mathbf{E}}\left\{\left[\left(1-\chi_{c}\right) \mathbf{I}_{4}+\chi_{c} \boldsymbol{\phi}_{c}\right] \mathbf{E}\right\},
$$

where all tensorial quantities are now reduced to scalar ones.

The fibers induce anisotropy in the composite behavior and, accordingly, the stresses are also functions of the stretching direction, characterized by the $\theta$ angle between the fibers and the stretching direction. Superscript $\theta$ in the second Piola-Kirchhoff stress $\mathbf{S}^{\theta}$ indicates the corresponding fiber direction.

An incremental form of Equation (15.8) suitable for solving the inverse problem can be obtained by considering a linearized stress-strain relationship over a finite time step; that is,

$$
\Delta \mathbf{S}^{\theta}=\Delta \mathbf{S}_{\mathrm{vol}}+\sum_{c=1}^{n} k_{c} \Delta \overline{\mathbf{S}}_{c}\left[\left(1-\chi_{c}\right) \mathbf{I}_{4}+\chi_{c} \boldsymbol{\phi}_{c}\right] .
$$


Similarly, the linearization of Equation (15.3) yields [20]

$$
\boldsymbol{\phi}_{c}=\left(\frac{\Delta \overline{\mathbf{S}}_{c}}{\Delta \mathbf{E}_{c}}\right)^{-1}:\left[\sum_{c=1}^{n} k_{c}\left(\frac{\Delta \overline{\mathbf{S}}_{c}}{\Delta \mathbf{E}_{c}}\right)^{-1}\right]^{-1} .
$$

The serial-parallel coupling parameter $\chi_{c}$ ranges between zero and one. If the stretch is applied in the fiber direction then the components exhibit a pure parallel behavior and $\chi_{c}=0$, while a pure serial case occurs when the stretch is perpendicular to the fiber direction, giving $\chi_{c}=1$. When the angle between the fibers and the stretching direction $\theta$ is different from $0^{\circ}$ or $90^{\circ}$, the composite response is a combination of serial and parallel behaviors. The value of $\chi_{c}$ in these intermediate cases is obtained as the ratio between the stiffness contribution of the fibers in the $\theta$ direction and the stiffness contribution of the fibers working in parallel.

A soft tissue considered as a biological composite material can be modeled as an isotropic matrix reinforced with long collagen fibers with preferred orientations [21]. Thereby, soft tissue can be considered in a simplified way as a collagen-reinforced composite. The amount of serial and parallel behavior of the whole composite depends on the angle between the fibers direction and the stretching direction. During a test, the fibers rotate; consequently, the corresponding angle is updated according to the change of configuration. The updated angle is obtained by means of a push-forward operation as

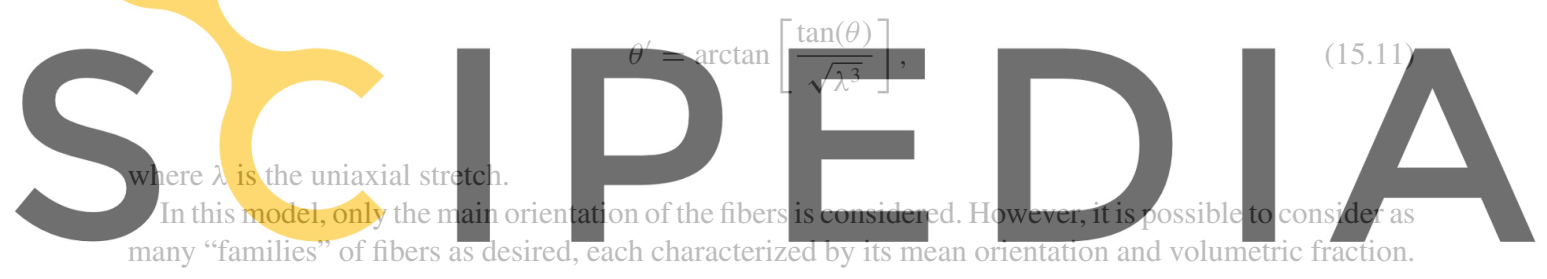

Register for free at https//www.scipedia.com to download the version without the watermark

\subsubsection{Definition of the Objective Function and Materials}

\section{Characterization Procedure}

As previously stated, soft tissue can be considered in a simplified way as a collagen-reinforced composite. Experimental tests of the main individual components of soft tissues are difficult. Therefore, the development of an analytical-numerical approach for the mechanical characterization of the main tissue components is very useful. In this chapter a constrained optimization problem is proposed and solved using experimental results.

The matrix main load-bearing components are elastin and a small amount of collagen fibers with random orientations. Owing to the presence of these collagen fibers, the matrix also exhibits a strong nonlinear behavior.

A hyperelastic neo-Hookean model is considered for the matrix and for the elastic contribution of the fibers. The goal of the optimization problem is to find the material parameters that best fit experimental data. The minimum experimental data necessary to carry out the numerical computations are two uniaxial tests of the tissue. Angle $\theta$ between the collagen fibers and the stretching direction must be different for each test, thus allowing one to asses the influence of the reinforcing orientation on the composite stiffness. The two tests are identified by means of these two angles termed $\theta_{1}$ and $\theta_{2}$.

The estimation of the material parameters requires the minimization of an objective function. The goal is the simultaneous minimization of the differences between the experimentally measured and the 
theoretically determined Cauchy stresses $\sigma$ for the two stretching directions $\theta_{1}$ and $\theta_{2}$ at each equilibrium configuration $k$ [21]. The objective function $R$ is defined as [1]

$$
R=\sum_{k=1}^{m}\left[\left(\left.\sigma_{\theta_{1}}\right|_{\mathrm{nu}}-\left.\sigma_{\theta_{1}}\right|_{\mathrm{ex}}\right)_{k}^{2}+\left(\left.\sigma_{\theta_{2}}\right|_{\mathrm{nu}}-\left.\sigma_{\theta_{2}}\right|_{\mathrm{ex}}\right)_{k}^{2}\right]
$$

where subscripts "ex" and "nu" denote experimental and numerical values, respectively, and $m$ is the number of equilibrium points considered.

The values of the material parameters are obtained from Equation (15.12) using a subspace trust region method based on the interior-reflective Newton method [22]. Upper and lower bounds for the material parameters are set for each constitutive model following Humphrey [21].

\subsubsection{Validation of the Inverse Model for Urinary Bladder Tissue Characterization}

The validation of the proposed model for characterization of the bladder tissue is presented next. The biological tissue is composed of a matrix of a mix of elastin, muscle, aqueous ground substance, and collagen fibers and it is considered to have an isotropic behavior. The matrix is reinforced by families of long collagen fibers with preferred orientations along the longitudinal direction of the bladder (i.e., oriented from the apex to the neck). The active behavior of the collagen and elastin bein Experimental data 1 fiber considering its ori et al. [17] using ECM results of uniaxial test
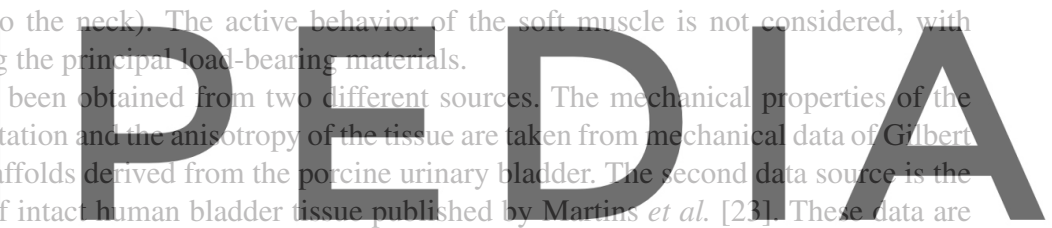

used to obtain the matrix properties of bladder tissue.

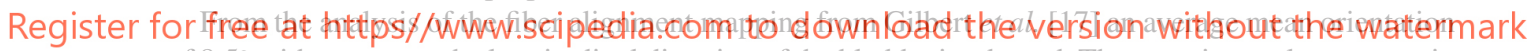

of $8.5^{\circ}$ with respect to the longitudinal direction of the bladder is adopted. The experimental stress-strain curves along the circumferential and longitudinal directions derived from Gilbert et al. [17] are shown in Figure 15.1. In these samples the fibers are more closely aligned with the longitudinal direction; consequently, the tissue stiffness is larger in that direction. The stress-strain relationships of the fibers are obtained by the application of the inverse method proposed using the ECM data shown in Figure 15.2. Most of the matrix components are removed during the scaffold preparation procedure. Consequently, a fiber participation ratio of 0.9 is adopted and the rest of the matrix takes the remaining volume. In order to simplify the resolution of the inverse problem, a fully imcompresible neo-Hookean model is considered for the fibers. The stress-strain relationship for the fiber obtained using this model is shown in Figure 15.2.

The fit of the ECM matrix obtained cannot be considered representative of the intact tissue matrix, and for this reason a second application of the inverse method is necessary. To this end, the data from the uniaxial test of intact urinary bladder tissue along its longitudinal direction are used (Figure 15.3). The data plotted are obtained by averaging the results corresponding to the uniaxial tests of human female bladder published by Martins et al. [23]. These tests correspond to 13 samples from female cadavers without observable clinical pelvic floor dysfunction with ages between 18 and 65 years. Consequently, they can be considered representative results for human bladder tissue.

In this second step the fiber stress-strain relationship is adopted from the results previously obtained and only the matrix model parameters are adjusted to fit the data from Figure 15.3. The stress-strain relationship of the human bladder matrix obtained is plotted in Figure 15.4. 


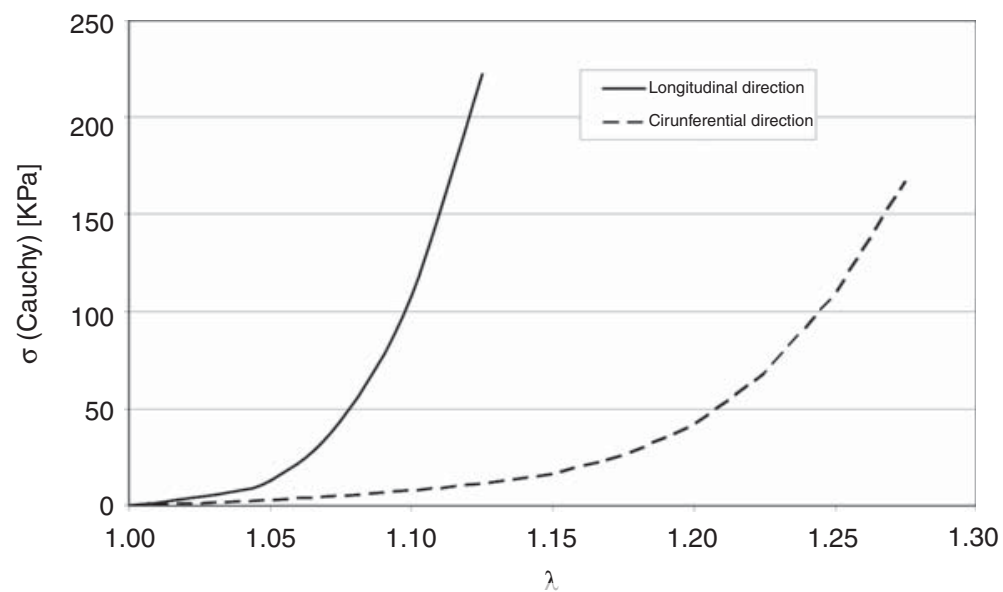

Figure 15.1 Average stress-strain relationship for the longitudinal and circumferential directions of ECM scaffolds derived from Gilbert et al. [17], with permission from Elsevier (C) 2008

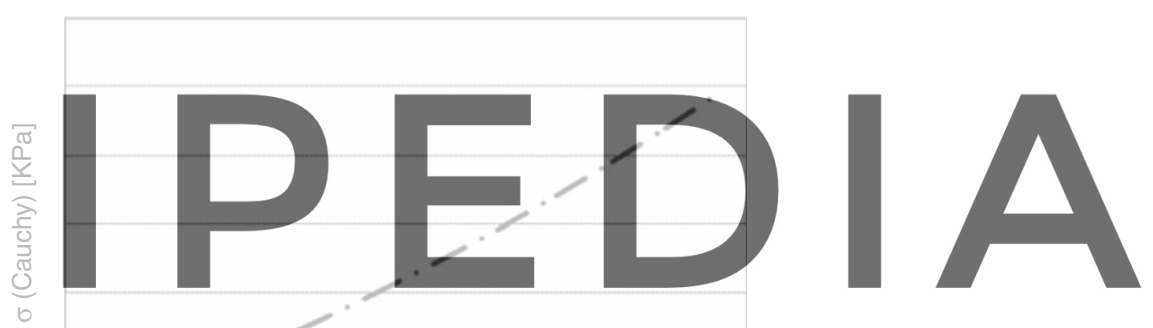

Register for free at https//Www.scipedia.com to download the version without the watermark

Figure 15.2 Stress-strain relationship obtained for the fiber fitting of experimental data from Gilbert et al. [17], with permission from Elsevier (C) 2008

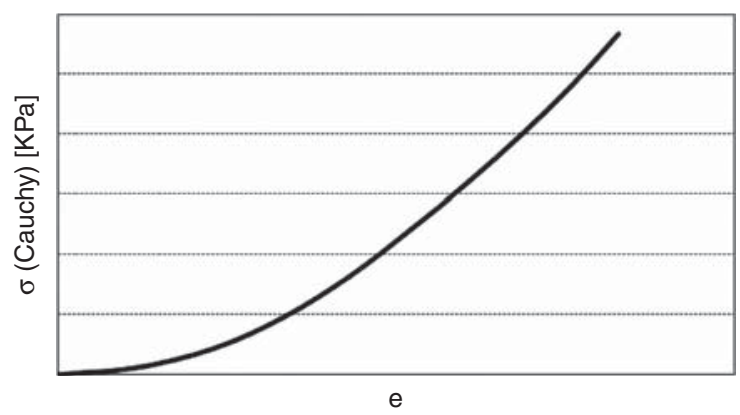

Figure 15.3 Averaged results of uniaxial tests along the longitudinal axis of human bladder tissue derived from Martins et al. [23], with permission from Springer (C) 2011 


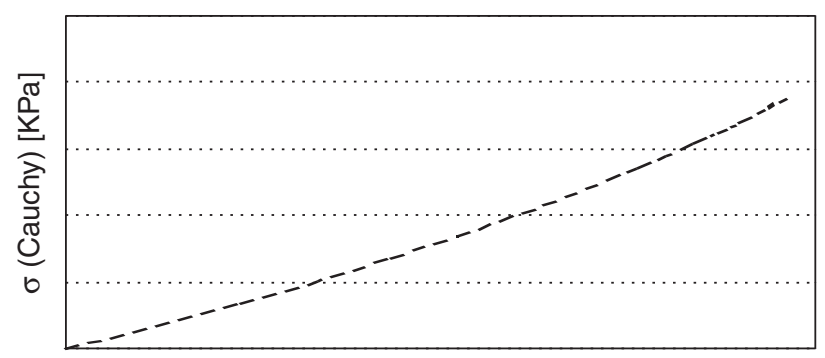

e

Figure 15.4 Stress-strain relationship obtained for the matrix fitting using experimental data from Martins et al. [23], with permission from Springer (C) 2011

This validation example shows that the procedure is suitable for the characterization of soft biological tissues modeled as fiber-reinforced composites. The methodology is flexible enough to be applied combining different sets of data, which is especially useful given the scarcity of experimental data regarding the mechanical behavior of soft-tissue components. In this first example, two components are just employed in the rule of mixtures; one component is the matrix, which is a mix of different materials, and the second component is constituted by the collagen fibers reinforcement. However, the rule of mixtures allows us to work with any number of components. Hence, a larger number of constituents

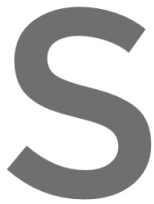
can be considered if the participation ratios, preferred or

\subsection{FEM Analysis of the Urinary Bladder}
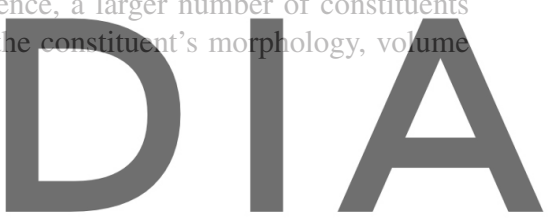

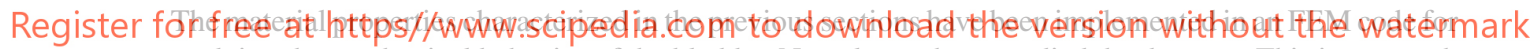

studying the mechanical behavior of the bladder. Namely, we have studied the detrusor. This is a smooth muscle which is responsible for maintaining an almost constant pressure inside the bladder during the filling and the storage of the urine [24].

Smooth muscles are known by their nonlinear behavior, and in the specific case of the detrusor the change in the stiffness is due not only to its mechanical properties but also to chemical reactions. The detrusor is innervated by an autonomic nervous system that allows the muscle to be partly contracted, maintaining tonus for prolonged periods with low energy consumption.

Given the complexity of biological materials and their multiscale hierarchy, some simplifications were made in the classical nonlinear continuum mechanics theory. The proposed model is based on the three-dimensional representation of the detrusor tissue by two different structures: a hyperelastic matrix, representing the extracellular substance, and viscoelastic fibers, representing the passive fibers [25]. For the sake of simplicity chemical reactions were not taken into account. Homogenization theory is used to represent the integration of these two main structures, whose resultant mechanical properties have been obtained from the rule of mixtures approach described in previous sections.

To represent the mechanics of the bladder, the constitutive equations and the kinematics are expressed in a total Lagragian description [26, 27].

The model considers the implementation of a hyperelastic-viscoelastic constitutive law into a finiteelement formulation accounting for fluid-structure interaction (FSI) [28] effects for the simulation of the deformation of the bladder wall as this is filled with urine. The resulting nonlinear FEM problem is solved by the Newton-Raphson method with a Bossak scheme. 


\subsubsection{Constitutive Model for Tissue Analysis}

The model is based on the representation of the detrusor tissue by two different structures: a hyperelastic matrix, representing the extracellular substance, and viscoelastic fibers, representing the passive fibers [29].

The phenomenological model describes the macroscopic nature of the materials as a continuum, and intends to represent the structure of the bladder tissue. The model is based on a hyperelastic neo-Hookean formulation. A subsequent extension of the formulation contemplates viscoelasticity effects and fiber behavior. The model has been validated using benchmark experiments [29]. Incompressibility is treated using the Ogden formulation for quasi-incompressible materials [30].

The neo-Hookean hyperelastic model was chosen with the following equations for the isochoric part of the strain energy potential and the stress respectively:

$$
\begin{aligned}
\Psi_{\text {iso }} & =\frac{1}{2} \mu\left(J^{-2 / 3} C_{k k}-3\right), \\
S_{i j \text { iso }} & =\frac{\partial \Psi_{\text {iso }}}{\partial E_{i j}}=\mu J^{-2 / 3}\left(\delta_{i j}-\frac{1}{3} C_{k k} C_{i j}^{-1}\right) .
\end{aligned}
$$

\section{The hyperelastic constitutive tensor matrix is obtained from}
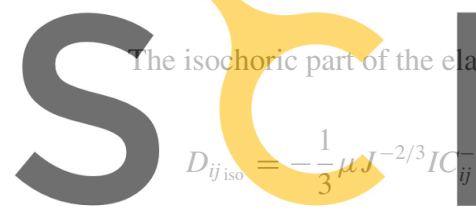

$$
\mathbb{D}=\frac{\partial \mathrm{S}}{\partial \mathbf{E}}=2 \frac{\partial \mathrm{S}}{\partial \mathbf{C}}=4 \frac{\partial^{2} \Psi}{\partial \mathbf{C} \partial \mathbf{C}}
$$

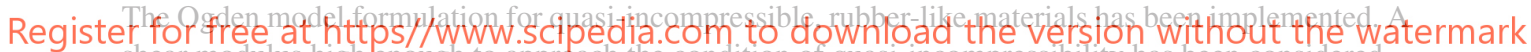 shear modulus high enough to approach the condition of quasi-incompressibility has been considered. \\ The volumetric part of the strain energy is given by}

$$
\Psi_{\mathrm{vol}}=k G(J) \quad \text { with } \quad G=\beta^{-2}\left(\beta \cdot \ln J+J^{-\beta}-1\right) \quad \text { and } \quad \beta=9 .
$$

The volumetric part of the stress is computed as

$$
\mathbf{S}_{\mathrm{vol}}=2 \frac{\partial \Psi_{\mathrm{vol}}(J)}{\partial \mathbf{C}}=J p \mathbf{C}^{-1} .
$$

The hydrostatic pressure $p$ and the volumetric part of the stress are obtained as

$$
p=\frac{\mathrm{d} \Psi_{\mathrm{vol}}(J)}{\mathrm{d} \mathbf{C}}=k \frac{\mathrm{d} G(J)}{\mathrm{d} J}, \quad \mathbf{S}_{\mathrm{vol}}=k \frac{1}{\beta}\left(1-\frac{1}{J^{\beta}}\right) \mathbf{C}^{-1}
$$

and the volumetric part of the elasticity tensor as:

$$
\mathbf{D}_{\mathrm{vol}}=\frac{\partial \mathbf{S}_{\mathrm{vol}}}{\partial \mathbf{E}}=2 \frac{\partial \mathbf{S}_{\mathrm{vol}}}{\partial \mathbf{C}}=4 \frac{\partial^{2} \Psi_{\mathrm{vol}}}{\partial \mathbf{C} \partial \mathbf{C}}
$$


After some algebra we obtain

$$
D_{i j \mathrm{vol}}=\frac{k}{2}\left(\frac{11}{2} J^{-5 / 2}-J^{-9}\right) C_{i j}^{-1} C_{i j}^{-1}+\frac{k}{2}\left(1-J^{-9}\right)\left(-\frac{1}{2} C_{i k}^{-1} C_{j l}^{-1}+C_{i l}^{-1} C_{j k}^{-1}\right) .
$$

The final form of the elasticity tensor is given by Equation (15.15) and Equation (15.19):

$$
\begin{aligned}
\mathbf{D}= & \mathbf{D}_{\mathrm{iso}}+\mathbf{D}_{\mathrm{vol}}, \\
D_{i j}= & -\frac{1}{3} \mu J^{-2 / 3} I C_{i j}^{-1}+\left[\frac{1}{9} \mu J^{-2 / 3}+\frac{k}{2}\left(\frac{11}{2} J^{-5 / 2}-J^{-9}\right)\right] C_{i j}^{-1} C_{i j}^{-1} \\
& +\left[-\frac{1}{3} \mu J^{-2 / 3}+\frac{k}{2}\left(1-J^{-9}\right)\right]\left(-\frac{1}{2} C_{i k}^{-1} C_{j l}^{-1}+C_{i l}^{-1} C_{j k}^{-1}\right) .
\end{aligned}
$$

The previous elastic model has also been extended to account for viscoelastic effects. The viscoelastic model is based on the generalized Maxwell model [29,31]. The kinematics of the viscoelastic body are represented by a superposition of a purely elastic body with a fixed reference configuration $\Omega_{0}$ and a Maxwell body with a moving reference configuration $\Omega_{0}^{v}$.

The thermodynamic process of the body is described by the deformation measures $J(\mathbf{X}, t), \overline{\mathbf{C}}(\mathbf{X}, t)$, and $\overline{\mathbf{C}}^{v}\left(\mathbf{X}_{v}, t\right)$ and the second-order structural tensor $\mathbf{A}(\mathbf{X})$.

For incompressible material, viscous effects only affect the isochoric part of the free energy, split into a thermodynamic part (pure hyperelastic deformation) and a dissipative part (viscoelastic response).

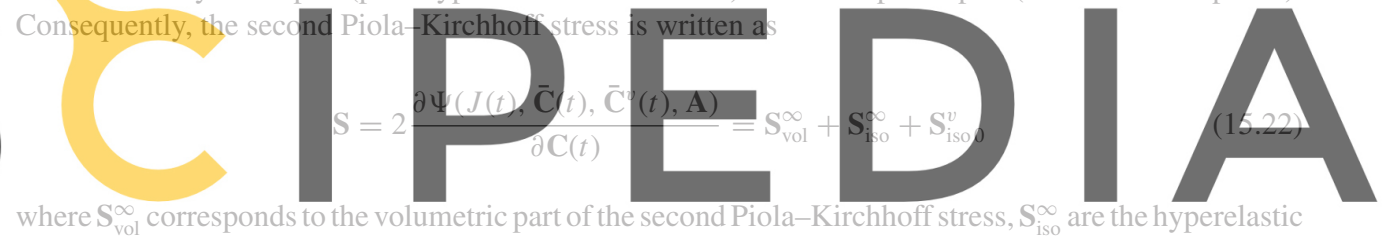

where $\mathrm{S}_{\mathrm{vol}}^{\infty}$ corresponds to the volumetric part of the second Piola-Kirchhoff stress, $\mathrm{S}_{\mathrm{is} 0}^{\infty}$ are the hyperelastic

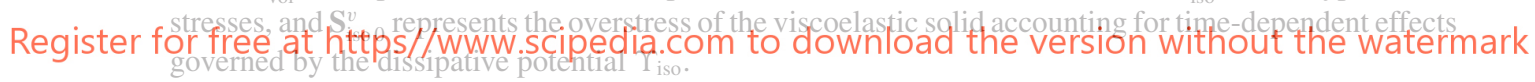

The different components of the second Piola-Kirchhoff stresses are computed as

$$
\mathbf{S}_{\mathrm{vol}}^{\infty}=2 \frac{\partial \Psi_{\mathrm{vol}}^{\infty}(J(t))}{\partial \mathbf{C}(t)}, \quad \mathbf{S}_{\mathrm{iso}}^{\infty}=2 \frac{\partial \Psi_{\mathrm{vol}}^{\infty}(\overline{\mathbf{C}}(t), \mathbf{A})}{\partial \mathbf{C}(t)}, \quad \mathbf{S}_{\mathrm{iso} 0}^{v}=2 \frac{\partial \Upsilon_{\mathrm{iso}}\left(\overline{\mathbf{C}}(t), \overline{\mathbf{C}}^{v}(t), \mathbf{A}\right)}{\partial \mathbf{C}(t)}
$$

The overstress of the viscoelastic solid reads

$$
\mathbf{S}_{\mathrm{iso} 0_{n+1}}^{v}=\xi_{n+1}^{2} \mathbf{S}_{\mathrm{iso} 0_{n}}^{v}+\beta \xi_{n+1}\left(\mathbf{S}_{\mathrm{iso}_{n+1}}^{\infty}-\mathbf{S}_{\mathrm{iso}_{n}}^{\infty}\right) .
$$

A more refined model for the detrusor was also developed by assuming a hyperelastic matrix reinforced with viscoelastic fibers. A random orientation of the fibers was considered. The model intends to represent the phenomenological behavior of the microstructure of the detrusor tissue, where the matrix consists of a neo-Hookean hyperelastic material and the collagen fibers are represented by two classes of perpendicular viscoelastic fibers at the element level. A similar model was proposed by Kondo et al. [32] using experiments to obtain the hyperelastic and viscoelastic parameters.

In order to account for the effect of fibers, the phenomenological model considers the second PiolaKirchhoff stress as a summation of the following tensors:

$$
\mathbf{S}=\mathbf{S}_{\mathrm{vol}}+\mathbf{S}_{\mathrm{iso}}^{\infty}+\mathbf{S}_{\mathrm{f}}
$$


where $\mathbf{S}_{\mathrm{vol}}$ and $\mathbf{S}_{\mathrm{iso}}^{\infty}$ are respectively the volumetric and the hyperelastic contributions of the stress, computed as described previously, and $\mathbf{S}_{\mathrm{f}}$ is the fiber contribution to the stress tensor, defined as

$$
\mathbf{S}_{\mathrm{f}}=\mathbf{S}_{\mathrm{f}}^{\infty}+\mathbf{S}_{\mathrm{f}}^{v}
$$

where $\mathbf{S}_{\mathrm{f}}^{\infty}$ corresponds to the stress contribution to the elastic part of the Maxwell model (the hyperelastic response described in Equation (15.22)) and $\mathbf{S}_{\mathrm{f}}^{v}$ corresponds to the viscous contribution of the Maxwell model.

Collagen fibers are considered by introducing the unit fiber direction $\mathbf{M}$ denoting the referential/local orientation of the fibers. The local structure of the material in the reference configuration $\Omega_{0}$ is defined by a symmetric second-order structural tensor $\mathbf{A}=\mathbf{M} \otimes \mathbf{M}$. The expression of this vector in the current configuration is $\mathbf{a}=\mathbf{F A F}^{\mathrm{T}}$.

Once fibers are accounted for, the material becomes anisotropic. We thus introduce a fourth invariant tensor $\mathbf{I}_{4}$ to represent the fibers orientation, defined as

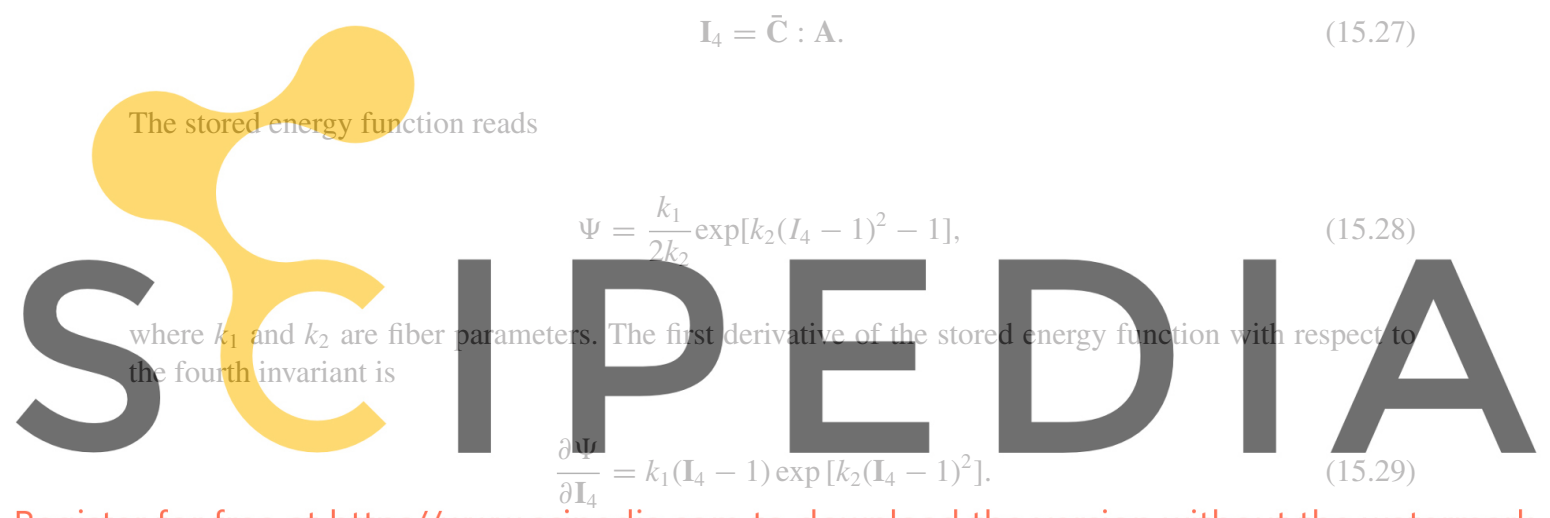

Register for free at https//www.scipedia.com to download the version without the watermark

Finally, the second Piola-Kircchoff stress contribution of the fiber is

$$
\mathbf{S}_{\mathrm{f}}=2 \frac{\partial \Psi\left(\mathbf{I}_{4}\right)}{\partial \mathbf{C}}=2 \frac{\partial \Psi}{\partial \mathbf{I}_{4}} \frac{\partial \mathbf{I}_{4}}{\partial \mathbf{C}}=2 J^{-2 / 3} \frac{\partial \Psi}{\partial \mathbf{I}_{4}}\left[\mathbf{A}-\frac{1}{3}(\mathbf{A}: \overline{\mathbf{C}}) \overline{\mathbf{C}}^{-1}\right] .
$$

The previous constitutive model was implemented into a standard finite-element formulation using both three-noded membrane triangles, rotation-free shell triangles, and stabilized four-noded tetrahedra to model the bladder wall. For details, see Monteiro [29].

\subsubsection{Validation. Test Inflation of a Quasi-incompressible Rubber Sphere}

The hyperelastic neo-Hookean model and the finite-element formulation were validated in the study of the inflation of a quasi-incompressible rubber sphere [29, 33, 34]. For the sake of simplicity, only a half-sphere was considered, accounting from symmetry (Figure 15.5).

The inflation pressure is a function of Cauchy stress and the radius and thickness of the sphere as

$$
p_{i}=2 \frac{h}{r} \sigma
$$



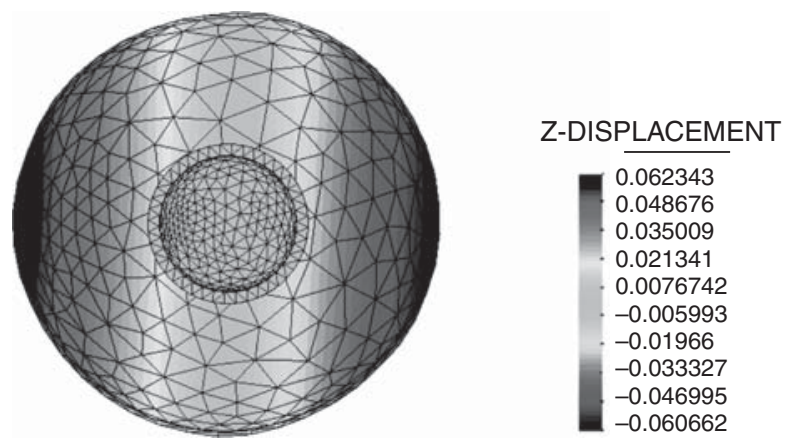

Figure 15.5 Results for the rubber sphere inflation. The internal half-sphere shows the initial geometry

Taking into account the incompressibility of the material, and using the constitutive equation, we can obtain the relation between the stretch in the principal direction (or circumferential stretch) and the associated circumferential Cauchy stress as

$$
\sigma=\mu_{\mathrm{p}}\left(\lambda^{\alpha_{\mathrm{p}}}-\lambda^{-2 \alpha_{\mathrm{p}}}\right)
$$

where $\alpha_{\mathrm{p}}=2$ and $\mu=\mu_{1}=624.0 \mathrm{kPa}$ is the shear modulus, previously represented by $C_{1}$ for the neo-Hookean model.

The geometrical data considered for the validation test are initial internal radius $0.03 \mathrm{~m}$ and initial thickness $0.005 \mathrm{~m}$. The finite-element mesh consists of 3278 four-noded tetrahedra and 675 nodes.

Figure 15.6 shows the results for evolution of the circumferential Cauchy stress versus the stretch $\lambda$, considering a bulk modulus of $10000 \mathrm{kPa}$. The analytical result for incompressible rubber is also shown.

The inflation of a half-sphere under internal pressure provides a first approximation of bladder inflation.

\subsubsection{Mechanical Simulation of Human Urinary Bladder}

Our goal is to simulate the human bladder under filling conditions. For this purpose, we start with the geometry from a simplified high-resolution three-dimensional model of the human bladder of a

\section{Stress $X \lambda$}

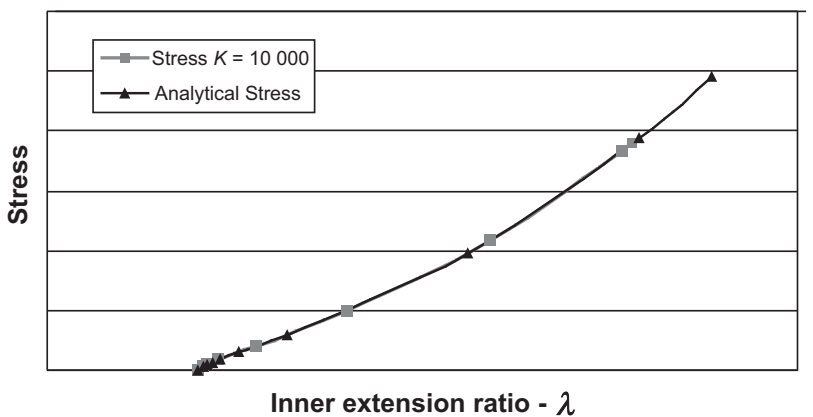

Figure 15.6 Circumferential Cauchy stress versus stretch $\lambda$ 


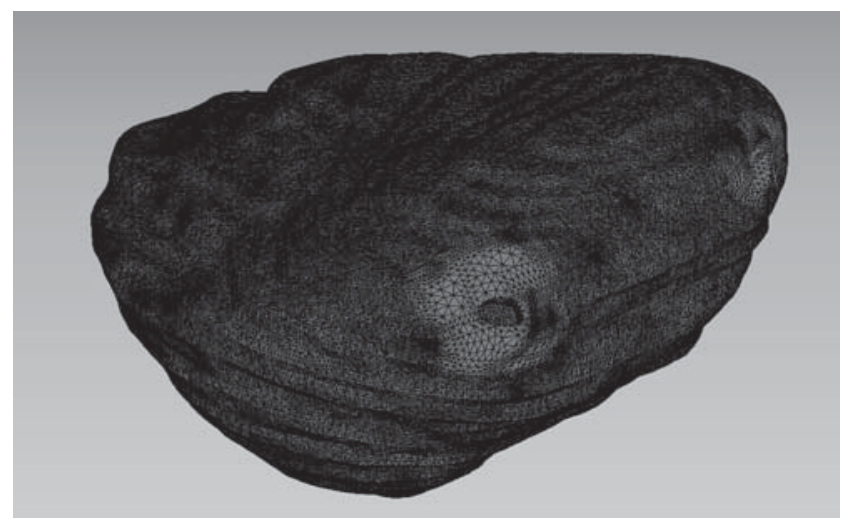

Figure 15.7 Three-dimensional model of the urinary bladder meshed with four-noded tetrahedra

39-year-old man with no known urological diseases [29]. The morphology was obtained from the Visible Human Project ( $\mathrm{VH})$ and the input geometry from the work of Tonar et al. [35]. The geometry was treated with the pre-post processor GID (www.gidhome.com). The initial mesh size discretizing the internal volume is of the order of 1 million four-noded tetrahedra (Figure 15.7).

In order to get a faster convergence and a reduced number of elements, the discretization of the bladder wall was simplified using three-noded membrane triangles (Figure 15.8).

The membrane formulation for the bladder wall was used in combination with the particle FEM (PFEM) [36-40] simulating the flow of urine during filling accounting for the bladder and urine interactions.

To simulate the filling of the bladder with urine, we start with a simple geometry considering only part of one ureter connected to a reservoir. Before proceeding to the simulation of bladder filling, boundary conditions are applied based on the study of the physiology and dynamics of the urinary apparatus and pelvic region. In the urinary bladder, the region corresponding to the trigone is considered to be fixed and zero displacements are imposed on this region (Figure 15.9). The surrounding lower part of the bladder also has restricted mobility to represent the contact with the pelvic musculature (Figure 15.10).
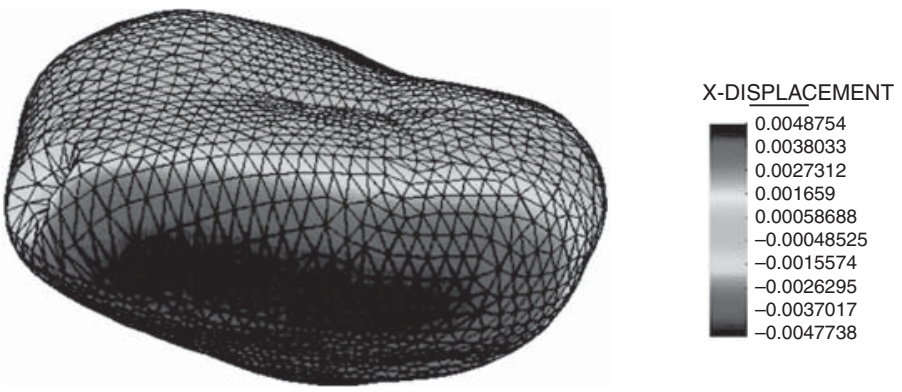

step 0.0023

Contour Fill of DISPLACEMENT, X-DISPLACEMENT.

Deformation (x1): DISPLACEMENT of Kratos, step 0.0023.

Figure 15.8 Urinary bladder wall meshed with three-noded membrane triangles 


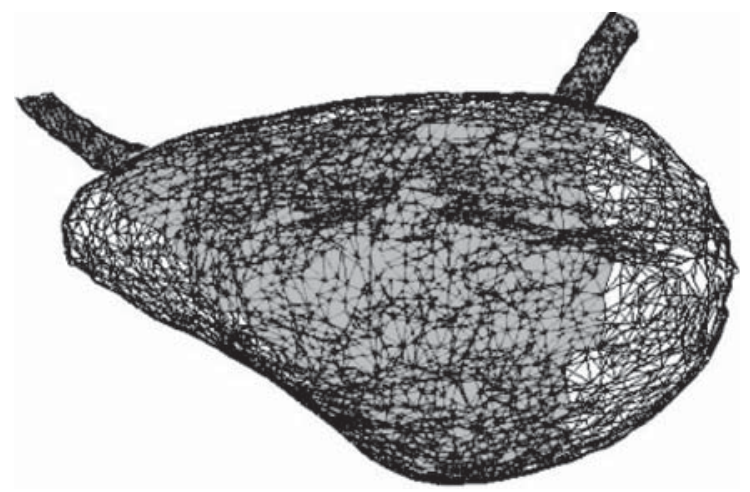

Figure 15.9 Zero displacement imposed on the trigone area (shaded)

The nonlinear viscoelastic constitutive model was tested first for the geometry of the bladder meshed with three-noded quasi-incompressible membrane elements. The simplified condition of zero displacement in the junction with the ureters was imposed. The initial internal pressure of $67 \mathrm{~Pa}$ corresponds to the maximum hydrostatic pressure within the $50 \mathrm{ml}$ bladder, computed with the PFEM. The pressure was then increased up to a maximum of $1000.0 \mathrm{~Pa}$, (or $10 \mathrm{~cm} \mathrm{H}_{2} \mathrm{O}$ ), which corresponds to the expected pressure of urine within the bladder for an average adult during filling conditions [38].

Figure 15.11 shows the deformed shape of the bladder at two filling instants.

\subsubsection{Study of Urine-Bladder Interaction}

The entrance of urine in the bladder was simulated by injecting fluid nodes at the extremity of each ureter. Figure 15.12 shows the mesh used for the bladder filling study accounting for urine-bladder interaction. In this simulation, the initial volume of the bladder, also known as residual volume, is of the order of $50 \mathrm{ml}$. Figure 15.13 shows the geometry of the bladder at two filling instants.

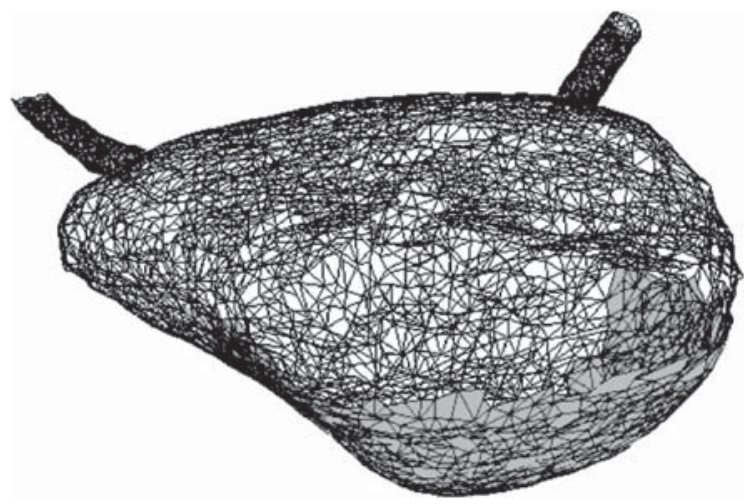

Figure 15.10 Restricted displacement imposed on the area in contact with pelvic musculature (shaded) 

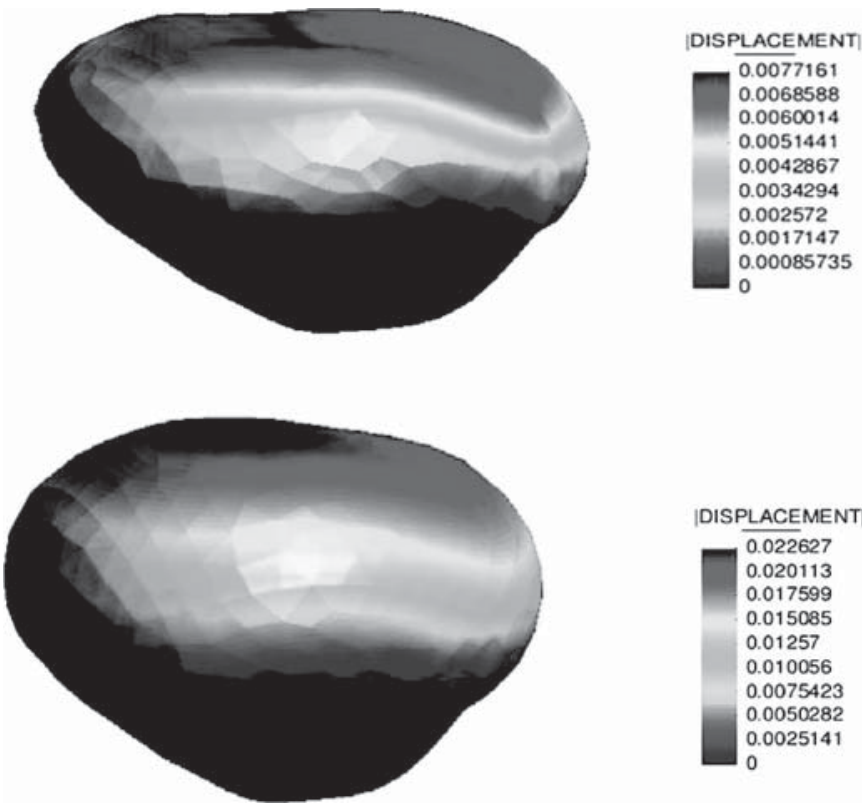

Figure 15.11 Bladder displacements with different volumes: $70 \mathrm{ml}$ (top) and $100 \mathrm{ml}$ (bottom)

Figure 15.14 shows the results for the Cauchy stresses at different points of the bladder wall for the numerical analysis considering the constitutive model with fibers. Results are compared with approximated expected values computed with the Laplace equation:

$$
T=P_{\text {ves }} \frac{R}{2 d}
$$

where $P_{\text {ves }}$ is the internal pressure, $d$ is current thickness, and $R$ is the current radius.

Figure 15.15 shows the results for the pressure-filling volume curve obtained accounting for urinewall interaction effects. Experimental results obtained with urodynamic data obtained in cystometry tests [24] are also shown for comparison.

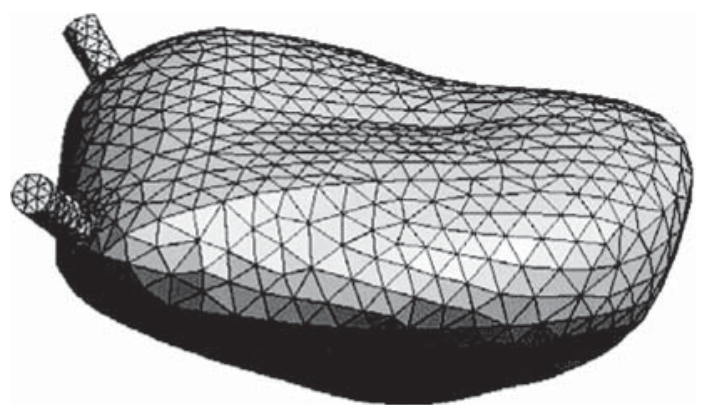

Figure 15.12 Mesh for bladder filling study 


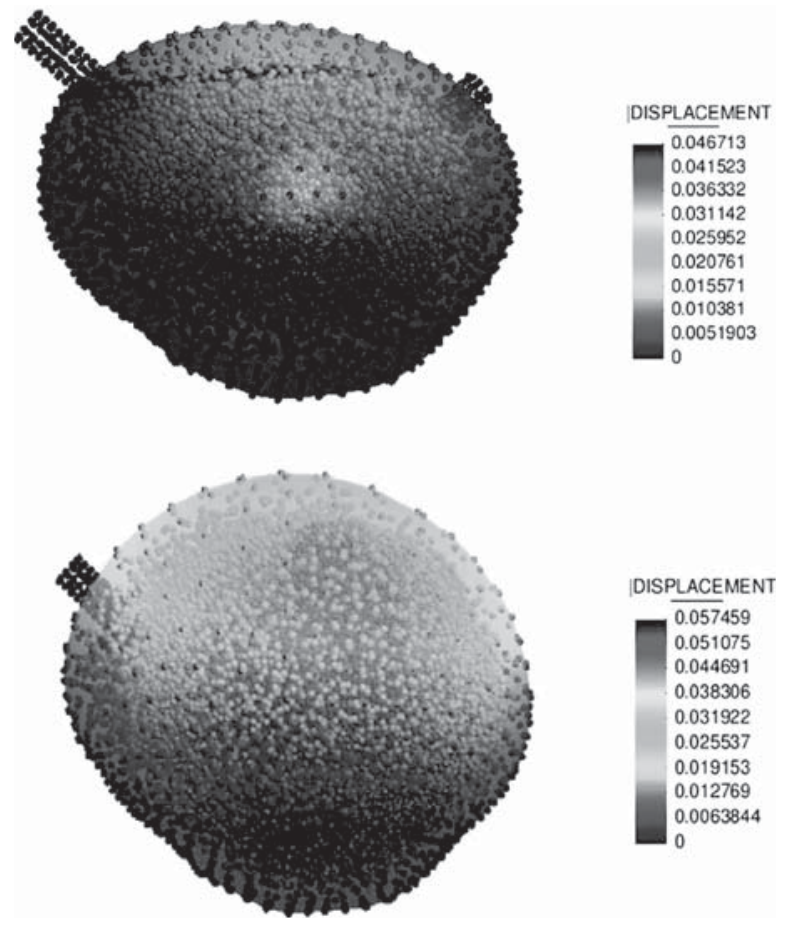

Figure 15.13 Displacement distribution and fluid nodes for filling volumes of $65 \mathrm{ml}$ (top) and $113 \mathrm{ml}$ (bottom)

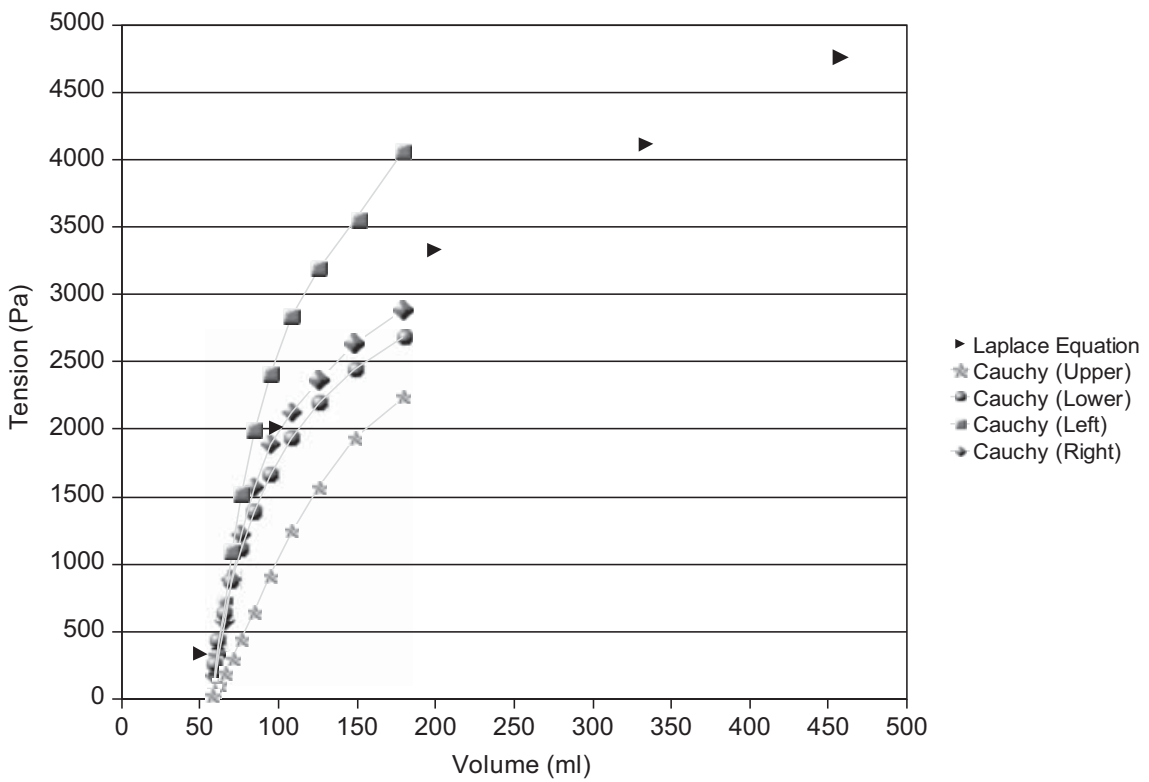

Figure 15.14 Principal Cauchy stresses versus volume (homogenized model, shear modulus $5 \mathrm{kPa}$ ) 


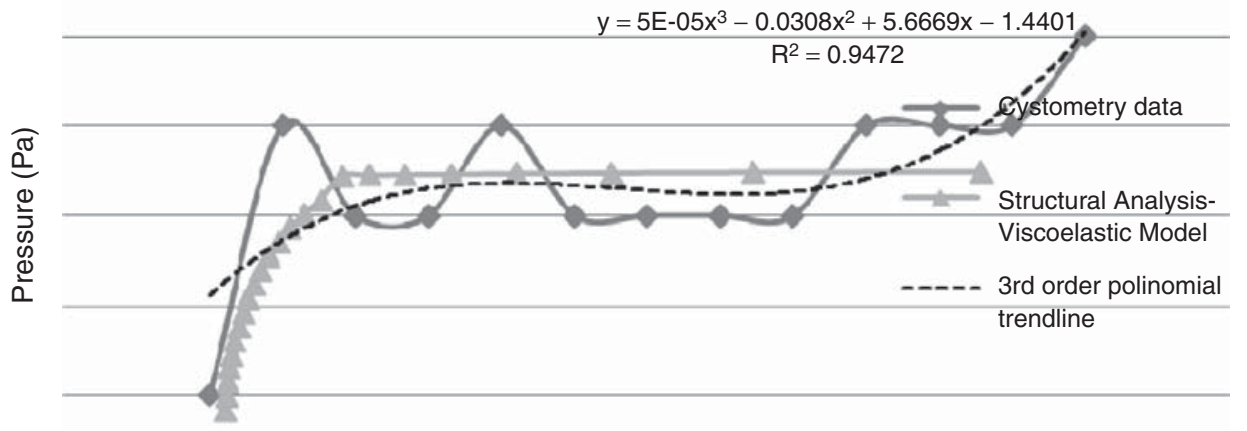

Volume (ml)

Figure 15.15 Comparison of pressure-volume results for the structural analysis of the bladder filling with experimental data obtained from urodynamic tests (cystometry) on a healthy patient. Dashed line shows the average of experimental results

\subsection{Conclusions}

An inverse method for determining the mechanical properties of the main components of soft tissues, starting from experimental stress-strain of a complete soft biological tissue, has been presented. The determination of the mechanical properties of tissue constituents is a necessary step for successfully modeling the behavior of the tissue as a reinforced composite material. The inverse problem proposed here has been applied to bladder tissue in two steps. First, we take advantage of the experimental information published by Gilbert et al. [17] regarding ECM scaffolds from bladder tissue. These results are used as input data for the inverse method to estimate the mechanical properties of the fibers. Once the mechanical properties of the fibers are estimated, the matrix properties of the bladder tissue are obtained by fitting experimental data from uniaxial tests of intact human bladder tissue [23].

The mechanical analysis of the bladder during filling has been studied with the FEM by considering a hyperelastic matrix reinforced with viscoelastic fibers. The results obtained are within the expected values for a normal human bladder.

\section{Acknowledgments}

We acknowledge the support of projects CAMBIO (MAT2009-10258), Ministerio de Ciencia e Innovación of Spain, AECID (A/024063/09), and AGAUR (Generalitat de Catalunya, Spain) for its economic support through the FI predoctoral grant.

\section{References}

[1] Bellomo, F.J., Oller, S., and Nallim, L.G. (2011) An inverse approach for the mechanical characterisation of vascular tissues via a generalised rule of mixtures. Computer Methods in Biomechanics and Biomedical Engineering, doi:10.1080/10255842.2011.585976.

[2] Vesely, I. (1997) The role of elastin in aortic valve mechanics. Journal of Biomechanics, 31, 115-123. 
[3] Roeder, B.A., Kokini, K., Sturgis, J.E. et al. (2002) Tensile mechanical properties of three-dimensional type I collagen extracellular matrices with varied microstructure. Journal of Biomechanical Engineering, 124, 214222.

[4] Holzapfel, G.A. (2008) Collagen in arterial walls: biomechanical aspects, in Collagen, Structure and Mechanics (ed. P. Fratzl), Springer-Verlag, Heidelberg, pp. 285-324.

[5] Fratzl, P., Misof, K., Zizak, I. et al. (1997) Fibrillar structure and mechanical properties of collagen. Journal of Structural Biology, 122, 119-122.

[6] Puxkand, R., Zizak, I., Paris, O. et al. (2002) Viscoelastic properties of collagen: synchrotron radiation investigations and structural model. Philosophical Transactions of the Royal Society B: Biological Sciences, 357, 191-197.

[7] Eppell, S.J., Smith, B.N., Kahn, H., and Ballarini, R. (2006) Nano measurements with micro-devices: mechanical properties of hydrated collagen fibrils. Journal of the Royal Society Interface, 3, 117-121.

[8] Shen, Z.L., Dodge, M.R., Kahn, H. et al. (2008) Stress-strain experiments on individual collagen fibrils. Biophysical Journal, 95, 3956-3963.

[9] Sacks, M.S. and Gloeckner, D.C. (1999) Quantification of the fiber architecture and biaxial mechanical behavior of porcine intestinal submucosa. Journal of Biomedical Materials Research, 46 (1), 1-10.

[10] Gloeckner, D.C., Sacks, M.S., Billiar, K.L., and Bachrach, N. (2000) Mechanical evaluation and design of a multilayered collagenous repair biomaterial. Journal of Biomedical Materials Research, 52 (2), 365-373.

[11] Gilbert, T.W., Sacks, M.S., Grashow, J.S. et al. (2006) Fiber kinematics of small intestinal submucosa under biaxial and uniaxial stretch. Journal of Biomechanical Engineering, 12 (6), 890-898.

[12] Atala, A., Bauer, S.B., Soker, S. et al. (2006) Tissue-engineered autologous bladders for patients needing cystoplasty. Lancet, 367, 1241-1246.

[13] Badylak, S.F., Vorp, D.A., Spievack, A.R. et al. (2005) Esophageal reconstruction with ECM and muscle tissue in a dog model. Journal of Surgical Research, 128 (1), 87-97.

[14] Bolland, F., Korossis, S., Wilshaw, S.P. et al. (2007) Development and characterisation of a full-thickness acellular porcine bladder matrix for tissue engineering. Biomaterials, 28 (6), 1061-1070.

[15] Brown, A.L., Farhat, W., Merguerian, P.A. et al. (2002) 22 week assessment of bladder acellular matrix as a bladder augmentation material in a porcine model. Biomaterials, 23 (10), 2179-2190.

[16] Kochupura, P.V., Azeloglu, E.U., Kelly, D.J. et al. (2005) Tissue-engineered myocardial patch derived from extracellular matrix provides regional mechanical function. Circulation, 30 (112, Suppl. 9), I144-I149.

[17] Gilbert, T.W., Wognum, S., Joyce, E.M. et al. (2008). Collagen fiber alignment and biaxial mechanical behavior of porcine urinary bladder derived extracellular matrix. Biomaterials, 29 (36), 4775-4782.

[18] Car, E., Oller, S., and Oñate, E. (2000) An anisotropic elasto-plastic constitutive model for large strain analysis of fiber reinforced composite material. Computer Methods in Applied Mechanics and Engineering, 185 (2-4), 245-277.

[19] Bellomo, F.J., Oller, S., Armero, F., and Nallim, L.G. (2011) A general constitutive model for vascular tissue considering stress driven growth and biological availability. CMES - Computer Modeling in Engineering and Sciences, 80 (1), 1-21.

[20] Bellomo, J.F. (2012) Numerical simulation of mechanical behavior of in vivo soft biological tissue. PhD thesis, Faculty of Engineering of National University of Salta, Argentina (in Spanish).

[21] Humphrey, J.D. (2002) Cardiovascular Solid Mechanics, Cells, Tissues and Organs, Springer.

[22] Coleman, T.F. and Li, Y. (1996). An interior, trust region approach for nonlinear minimization subject to bounds. SIAM Journal on Optimization, 6, 418-445.

[23] Martins, P.A., Filho, A.L., Fonseca, A.M. et al. (2011) Uniaxial mechanical behavior of the human female bladder. International Urogynecology Journal, 22 (8), 991-995.

[24] Wein, A., Kavoussi, L., Novick, A. et al. (2007). Campbell-Walsh Urology, 9th edition, Saunders.

[25] Cimrman, R. (2005) Mathematical modelling of biological tissues. PhD thesis, University of West Bohemia.

[26] Holzapfel, G.A. (2000) Nonlinear Solid Mechanics: A Continuum Approach for Engineering, John Wiley \& Sons.

[27] Belytschko, T., Liu, W.K., and Moran, B. (2000) Nonlinear Finite Elements for Continua and Structures, John Wiley \& Sons.

[28] Idelsohn, S., Del Pin, F., Rossi, R., and Oñate, E. (2009) Fluid-structure interaction problems with strong added mass effect. International Journal for Numerical Methods in Engineering, 80 (10), 1261-1294.

[29] Monteiro, V.S.A. (2012) Computational model of the human urinary bladder. PhD thesis, Department of Strength of Material and Structures of Technical University of Catalonia (Barcelona Tech. Spain). 
[30] Ogden, R.W. (1984) Non-Linear Elastic Deformations, Ellis Horwood Ltd.

[31] Holzapfel, G. and Gasser, C. (2001) A viscoelastic model for fiber-reinforced composites at finite strains: continuum basis, computational aspects and applications. Computer Methods in Applied Mechanics and Engineering, 190, 4379-4403.

[32] Kondo, A., Susset, J., and Lefaivre, J. (1972) Viscoelastic properties of bladder. I. Mechanical model and its mathematical analysis. Investigative Urology, 10 (2), 154-163.

[33] Treloar, L.R.G. (1944) Stress-strain data for vulcanised rubber under various types of deformation. Transactions of the Faraday Society, 40, 59-70.

[34] Alexander, H. (1971) Tensile instability of initially spherical balloons. International Journal of Engineering Sciences, 9, 151-162.

[35] Tonar, Z., Zátura, F., and Grill, R. (2004) Surface morphology of kidney, ureters and urinary bladder models based on data from the visible human male. Biomedical Papers of the Medical Faculty of the University of Palacky Olomuc Czech Republic, 148 (2), 249-251.

[36] Oñate, E., Idelsohn, S.R., Del Pin.F., and Aubry, R. (2004) The particle finite element method, an overview. International Journal of Computational Methods, 1 (2), 267-307.

[37] Oñate, E., Idelsohn, S.R., Celigueta, M.A., and Rossi, R. (2008) Advances in the particle finite element method for the analysis of fluid-multibody interaction and bed erosion in free surface flows. Computer Methods in Applied Mechanics and Engineering, 197, 1777-1800.

[38] Chapple, C.R. and MacDiarmid, S.A. (2000) Urodynamics Made Easy, 2nd edition, Churchill Livingstone, Philadelphia, PA.

[39] Rossi, R. (2005). Light-weight structures: structural analysis and coupling issues. PhD thesis, University of Padova.

[40] Rossi, R., Ryzhakov, P.B., and Oñate, E. (2009) A monolithic FE formulation for the analysis of membranes in fluids. International Journal of Spatial Structures, 24 (4), 205-210. 